\title{
Escritura inicial y estilo cognitivo
}

\section{Initial Writing and Cognitive Style}

\author{
Lida Johana Rincón Camachor \\ Christian Hederich Martínez ${ }^{3}$
}

\section{Resumen}

Se examinaron los efectos comparados de dos métodos para la enseñanza de la escritura en el nivel inicial: global y silábico y su interacción con el estilo cognitivo en la dimensión de dependencia-independencia de campo (DIC), en 31 niños de dos grupos escolares de primer grado de primaria, de dos instituciones diferentes, en donde cada uno de los métodos de venían implementando. Se aplicaron la prueba CEFT para la determinación del estilo cognitivo y la prueba de escritura "ejercicio de creación de texto" al inicio del año escolar del primer grado y cuatro meses después. Los resultados finales fueron examinados a través de análisis de covarianza en los que se controlaron, como covariables, los logros registrados cuatro meses antes. Los datos muestran evidentes ventajas del método global sobre el método silábico en términos generales, y muy específicamente para los niños de estilo dependiente de campo o intermedio en seis de los ocho los indicadores examinados. Estos resultados son explicados a partir de características distintivas del estilo cognitivo en esta dimensión.

Palabras clave:

Aprendizaje de la escritura, método global, método silábico, estilo cognitivo, capacidad de atención.

\section{Abstract}

Comparative effects of two methods used for teaching writing at the initial level were examined: the global and syllabic approaches and their interaction with cognitive style in the field dependence-independence dimension (FDI), in 31 first-grade children from two school groups and from two different institutions, where each one of the methods were implemented. CEFT test was applied to determine the cognitive style, and a writing test called "text-building exercise" was also used at the beginning of the academic year and four months later. The final results were esamined through covariance analysis, in which the achievements recorded four months earlier were controlled as covariates. In general, data show clear advantages of the global method over the syllabic method, specifically for field-dependent and intermediate style children in six out of eight indicators examined. These results are explained on the basis of distinctive features of the cognitive style in this dimension.

\section{Keywords:}

Learning of writing, global method, syllabic method, cognitive style, attention capacity.

Artículo recibido el 15 Enero de 2012 y aprobado el 30 Marzo de 2012

1 Este artículo presenta algunos de los resultados del proceso de investigación "Relaciones entre el aprendizaje de la lengua escrita, métodos de enseñanza y estilos cognitivos", financiado por el Centro de Investigaciones de la Universidad Pedagógica Nacional durante el año 2009 (véase Giraldo, 0.; Pérez; C. y Rincón, L; (2011), en las referencias).

2 Magister en Educación de la Universidad Pedagógica Nacional. Profesora de la Universidad Pedagógica Nacional. Grupo de Investigación en Estilos Cognitivos. ljrincon@pedagogica.edu.co

3 Doctor en Psicología de la Universidad Autónoma de Barcelona. Profesor de la Universidad Pedagógica Nacional. Grupo de Investigación en Estilos Cognitivos hederich@pedagogica.edu.co 


\section{Introducción}

En el marco de estudio de los procesos de aprendizaje de la escritura, surge el interés de explorar las relaciones entre los métodos de enseñanza de la escritura y las tendencias cognitivas de los sujetos al procesar la información, para reconocer sus efectos en los procesos de aprendizaje. El interés de este estudio responde, en principio, al propósito de presentar una explicación cognitiva de los procesos de aprendizaje de la escritura que permita identificar la pertinencia de las propuestas educativas planteadas alrededor de este tópico.

De este modo, el desarrollo de la propuesta de investigación se fundamenta en el reconocimiento, por una parte, de una perspectiva cognitiva de la escritura, entendiendo que esta requiere de conductas cognitivas como la planificación, la organización, la estructuración y la revisión; por otra parte, de las tendencias cognitivas reconocibles que poseen los sujetos y que intervienen en la forma como aprenden y, en general, cómo procesan la información; y, en tercer lugar, de las características de los métodos de enseñanza de la escritura que poseen requerimientos cognitivos diferentes. A partir de estas premisas, no resulta irracional pensar que cada método puede favorecer el aprendizaje de la lengua escrita en los sujetos según su tendencia estilística. Por tal razón, el objetivo de este estudio es explorar el efecto diferencial de la implementación de dos métodos para la enseñanza de la escritura (silábico y global), sobre el logro de aprendizaje en niños con estilos cognitivos diferentes en la dimensión de dependencia-independencia de campo (DIC).

Identificar las relaciones que se establecen entre factores individuales y factores ambientales que intervienen en el logro de aprendizaje de los individuos es una tarea compleja. Demanda el reconocimiento de un sinnúmero de variables que permiten caracterizar el comportamiento, no solo socio - afectivo sino cognitivo de los sujetos. Varios estudios en el ámbito educativo, han explorado la teoría de los estilos cognitivos con el propósito de comprender las relaciones que se establecen entre las diferencias individuales de los sujetos y el desempeño académico, en diferentes áreas de conocimiento. Del mismo modo, se ha explorado la relación entre los diferentes métodos y estrategias de enseñanza con los estilos cognitivos, especialmente en la dimensión de dependencia - independencia de campo.

De acuerdo con lo que compete a este estudio, se revisará, por una parte, el origen de los métodos de enseñanza de la escritura, sus fundamentos psicológicos y el concepto implícito que cada uno guarda sobre la escritura. Por otra parte, la teoría de los estilos cognitivos, en la dimensión de dependencia - independencia de campo y su relación con procesos de enseñanza - aprendizaje. Y finalmente, los antecedentes teóricos del estudio de la relación entre los métodos de enseñanza, el aprendizaje de la lengua escrita y los estilos cognitivos.

\section{Los métodos de enseñanza}

Existe una amplia discusión alrededor de los métodos que se consideran más pertinentes para abordar los procesos de adquisición del código escrito durante la etapa de la infancia. Por una parte, tales procesos de adquisición han sido entendidos como una simple transferencia del código oral a la representación gráfica del mismo (Benveniste, 1998), y por otra parte, se ha explicado que estos implican ciertos niveles de planificación y procesamiento cognitivo (Fayol y Schneuwly, 1986; citado en Jollibert, 2000).

En el ámbito educativo se ha hablado de dos grandes grupos de métodos, que han surgido con el propósito de satisfacer diferentes necesidades en el aprendizaje, de este modo, “...cada uno de ellos responde a las direcciones psicológicas dominantes en la época de su aparición y desarrollo y a sus respectivas interpretaciones del proceso del aprendizaje" (Braslavsky, 1962, 16). El primer grupo se conoce como "Métodos de marcha sintética", que presenta un desarrollo de la adquisición del código escrito a partir del estudio de los signos o de los sonidos elementales (grafías y fonemas) (Braslavsky, 1962, 24). El segundo grupo se conoce como "Métodos de marcha analítica", que propone iniciar el proceso a partir del reconocimiento de las palabras como un todo. 
Como se ha dicho, cada grupo de métodos está fundamentado en un paradigma psicológico particular, que determina la forma como se concibe al individuo que aprende. En lo que respecta al método sintético, han surgido controversias relacionadas con los posibles supuestos psicológicos que le sustentarían. Por un lado, algunos autores han afirmado que sus principios demuestran una carencia de sustento psicológico y un desconocimiento de los procesos de aprendizaje, en particular los asociados con la adquisición del código escrito. Por otro lado, se afirma que este, en cierta manera, busca responder a un concepto "precientífico del atomismo, para el cual los conocimientos, como todos los hechos de la vida psíquica, comienzan por sensaciones elementales", que luego se reúnen y vuelven a asociarse a través de procesos superiores (Braslavsky, 1962, 42). Desde esta mirada, cada método (alfabético, fonético y silábico) que aparece en este marco, inicia su instrucción a partir de lo que se conciba como el elemento o la unidad mínima, ya sea la letra, el fonema o la sílaba, para luego conducir al sujeto hacia un proceso de asociación que le permita integrar esos elementos en una unidad mayor y más compleja: la palabra.

Desde el punto de vista psicolingüístico, es posible sustentar este enfoque en dos direcciones. La primera, asociada con la explicación de que para comprender el código escrito, los individuos siguen una "ruta indirecta", y la segunda, relacionada con la teoría de procesamiento de "abajo - arriba".

Para explicar el concepto de "ruta indirecta", algunos estudios de la psicología cognitiva han demostrado que durante el procesamiento de palabras escritas, la información que es percibida a través de la vista es modificada, de forma que se presenta una transformación del código grafémico a otro, basado en el conocimiento adquirido en el habla, es decir, acústico y fonético. La postura que afirma que los lectores hacen, sin falta, este proceso de conversión, también se le conoce como recodificación (Best, 2004 y López y Álvarez, 1991).

Con respecto a la teoría de procesamiento de abajo - arriba, se dice que durante el proceso de la comprensión escrita inicia con la percepción de los elementos (letras), luego palabras y avanza a otras unidades de significado que implican procesos asociativos más complejos. Esto podría estar asociado con la capacidad cognitiva de los individuos de automatizar procesos tales como el reconocimiento de unidades mínimas y que facilitaría el alcance de otros niveles que demanden mayor control sobre el procesamiento. En este sentido, el método sintético se apoyaría en la premisa de que los individuos deberían iniciar el aprendizaje del código por el reconocimiento de las letras y su correspondencia con el conocimiento fonético - fonológico, para que una vez automatizado, se avance a las siguientes etapas.

Por su parte, el método analítico, tal y como lo define Braslavsky (1962), se fundamenta en un paradigma psicológico basado en el concepto de globalización, la percepción visual como actividad dominante, la lectura de ideas y la naturaleza del desarrollo cognitivo del individuo.

Con respecto al concepto de la globalización, y para explicar la naturaleza del desarrollo cognitivo de los individuos, es preciso señalar que desde una mirada cognitivista la percepción de los niños se caracteriza por una "visión de conjunto". En el caso específico de procesos lingüísticos, Karmiloff Smith $(1994,63)$ afirma que "el espacio de hipótesis que el niño se plantea respecto al significado de las palabras está sujeto a restricciones de principio. Esto es producto de un sesgo de dominio específico y de una sensibilidad a las distinciones que operan dentro del propio sistema lingüístico". De este modo, estudios como los adelantados por Markman (1990) demuestran que niños, incluso desde los 18 meses, pueden regirse por una serie de suposiciones relativas a las relaciones entre palabras y referentes, dentro de las que se haya la suposición del objeto completo, que actúa como sesgo probabilístico que facilita los procesos de adquisición de conceptos, guiando al individuo hacia una comprensión del objeto como un todo.

Del mismo modo, algunas aproximaciones a la descripción de los procesos naturales de adquisición del código escrito han permitido, en primer lugar, comprender que los niños están predispuestos cognitivamente a interactuar con el código mucho 
antes de su ingreso a la escuela, y en segundo lugar, identificar una serie de etapas que todos los niños atraviesan en el proceso. Emilia Ferreiro (1983) propone tres momentos de construcción de hipótesis por los que los niños se conducen hasta acceder completamente al código convencional: el primero es el de distinción entre dibujo - grafía, el segundo es el de control de cantidad y calidad y finalmente, el nivel de relación entre sonido y grafía.

Con relación a la percepción visual como actividad dominante, esta se sustenta en la explicación psicolingüística de "acceso directo" en la tarea de reconocimiento de palabras. En este caso, "las palabras son unidades "privilegiadas" de identificación en el transcurso de la lectura. Esto equivale a decir que las unidades léxicas del texto han de ser necesariamente descifradas o identificadas para poder comprender el lenguaje escrito, sin que sea prescindible, en cambio, la identificación exhaustiva de unidades inferiores a la palabra". (Belinchón, Igoa y Riviere, 2000, 350).

Así mismo, el "modelo de logogén" propuesto por Morton (1979) plantea que el proceso de recuperación de significado de las palabras no requiere de etapas, sino se da a través de la activación, por una parte, de unidades léxicas que concuerdan con los rasgos del estímulo percibido y por otra parte, de la información proporcionada por el contexto sintáctico y semántico de este. De esta manera, el proceso de activación se da por medio de dos vías que interactúan: la entrada sensorial y el sistema cognitivo. En esta misma dirección, lo que propone el método global, es que el individuo recibe el estímulo a través de la percepción visual, que entraría directamente a interactuar con la información del sistema cognitivo que contiene la información lexical, sin necesidad de traducir la señal visual (grafías) a su equivalente auditivo (fonemas).

A partir de la explicación sobre el dominio de la percepción visual, se puede comprender el concepto de lectura de ideas. El método global apunta a que el individuo no decodifique elementos aislados sin sentido, como lo serían en este caso las grafías, los fonemas o la sílabas, sino que comprenda unidades cargadas con un significado completo, desde la palabra hasta el texto en su totalidad.

\section{Estilo cognitivo}

El concepto de estilo cognitivo ha sido estudiado por diferentes escuelas psicológicas, que le han definido desde varias perspectivas. Messick (1984,w 59- 74) presenta tres orientaciones de su definición: 1] como diferencias individuales consistentes en las propiedades de la estructura cognitiva; 2] como modos característicos auto-consistentes de percibir, recordar, pensar y resolver problemas; o 3] como preferencias cognitivas o modos característicos en los que las personas prefieren conceptualizar y organizar el mundo de los estímulos.

En este estudio se adoptará el concepto de estilo cognitivo como modo característicos y autoconsistentes de la cognición que determinan maneras habituales de procesar información, que se traslucen en la realización de tareas concretas (Hederich, 2007, 24). En este sentido, en tanto el estilo cognitivo permite establecer características distintivas entre las personas, se hace conveniente determinar las formas en que este afecta los procesos de enseñanza y aprendizaje en el aula y para ello el presente trabajo centra su atención en la dimensión dependencia-independencia de campo (DIC), la cual hace referencia a "la tendencia de una persona a asignarle una organización y una estructura propias a la información disponible para realizar una tarea o resolver un problema, con independencia de la forma como tal información ha sido presentada, o en contraste, la tendencia a resolver la tarea o el problema manejando la información disponible sin desprenderla del contexto en que ha sido presentada y sin cambiarle su estructura y organización iniciales" (Hederich y Camargo, 2001, 8).

Desde la perspectiva cognitiva en la que se sustenta este estudio es evidente la importancia que el estilo cognitivo tiene en la dimensión dependencia-independencia de campo, en relación con el aprendizaje y la realización de tareas y la resolución de problemas como medios para acceder a este. Además, representa significativas implicaciones educativas, por cuanto permite diferenciar las formas de pensar y de 
dar respuesta a eventos, situaciones e informaciones que circulan en el ámbito escolar. Por ello resulta pertinente establecer algunas diferencias entre los individuos dependientes de campo (o también llamados sensibles al medio) e independientes de campo y comprender mejor, desde esta perspectiva, el marco en el que se sustentan los hallazgos de la presente investigación.

En primer lugar, desde la perspectiva del aprendizaje, los individuos sensibles al medio se presentan como individuos que "tienden a observar una situación como un todo; son capaces de mantener una perspectiva global y de apreciar las situaciones en su contexto general. Por su parte, los individuos independientes se presentan como individuos propensos a observar las situaciones como una colección de partes relativamente aisladas entre sí y con frecuencia focalizan su atención en uno o dos aspectos de la situación con exclusión de los otros" (Hederich y Camargo, 2001, 11). Estas características de los individuos dan cuenta de la necesidad de considerar en el aula de clase la manera en que el maestro presenta la información y el tipo de tarea que sugiere al estudiante para reforzar el proceso de aprendizaje de dicha información; así mismo, permite establecer diferencias en la forma en que el individuo tiende a dar respuesta a la tarea, condicionando el nivel de logro del aprendizaje.

En segundo lugar, y desde la perspectiva de la construcción de conceptos, los individuos sensibles "construyen conceptos a partir de sucesivas reformulaciones de una información inicial modificando la estructura del concepto original múltiples veces, adaptando la información que representa el concepto en su forma más general y abstracta. El individuo independiente, por su parte, complejiza su sistema conceptual probándolo y contrastándolo permanentemente con la realidad externa de la cual abstraen los elementos objetivamente relevantes, en independencia del contexto en que estos se encuentran" (Hederich y Camargo, 2001, 12). Esta tendencia en los individuos hace posible pensar en la relevancia que el aprendizaje inicial de la escritura tiene en relación con el estilo cognitivo, pues la escritura se constituye en una forma de reestructuración que implica entre otras operaciones, la combinación de letras, la conformación de palabras, la construcción de oraciones, la construcción de párrafos y textos completos con sentido.

Ahora bien, en relación con el trabajo en grupo, los individuos independientes "establecen una clara separación entre ellos y su entorno, incluidas las personas que lo rodean, lo que los hace autónomos e independientes, con tendencia a manejar sus relaciones sociales sobre la base de objetivos impersonales más que sobre la base de aspectos como la adscripción al grupo o la empatía personal. Por su parte, los sensibles al medio son personas que se perciben y definen a sí mismas como parte de un todo que los abarca. En esta medida, no establecen límites claros entre ellos y su entorno físico y social. Son individuos adscriptivos y sociables, con tendencia a manejar sus relaciones interpersonales sobre la base de niveles de empatía personal más que sobre la base de objetivos de trabajo" (Hederich y Camargo, 2001, 13). Si consideramos la relativa importancia que reporta al aprendizaje inicial de la escritura la interacción con otros, es posible desde esta caracterización determinar la incidencia del método escogido para dicho aprendizaje, pues según el individuo posea un estilo u otro, responderá mejor a uno u otro método sea este de enfoque sintético o analítico.

\section{Antecedentes}

Se han identificado varios estudios referidos a cómo los sujetos perciben y procesan la información, teniendo en cuenta características propias, que involucran aspectos biológicos, cognitivos y culturales, lo que se ve reflejado en procesos de aprendizaje singulares. Sin embargo, es poca la información de estudios que se hayan hecho alrededor de las implicaciones o efectos de la aplicación de métodos para la enseñanza de la escritura, teniendo en cuenta las diferencias individuales de los sujetos, específicamente, el estilo cognitivo en la dimensión dependencia-independencia de campo (DIC).

Rincón y Hederich (2008) constituyen el antecedente más reciente de este objeto de estudio, en tanto abordan la discusión entre las características cognitivas individuales de los estudiantes, sus modos 
de aprendizaje y los métodos de enseñanza. Así, los autores en su investigación examinan la relación entre el estilo cognitivo de los niños, los métodos de enseñanza y los niveles de desempeño en los procesos de aprendizaje de la lengua escrita. El interés del estudio parte de la necesidad de identificar las diferencias en la dimensión DIC de dos grupos de estudiantes de primeros grados de escolaridad, a fin de determinar el efecto diferencial de la aplicación de dos enfoques metodológicos distintos para la enseñanza de la escritura (silábico y global), sobre su aprendizaje en niños con estilos cognitivos diferentes, para lo cual se planteó una investigación de tipo pre-experimental pre-postest.

$\mathrm{Al}$ examinar la correlación entre postest y estilo cognitivo, para el caso del grupo de método silábico, se evidenció cómo este método favoreció en mayor medida al grupo de los sujetos independientes de campo en comparación con los sujetos de estilo cognitivo intermedio y sensible, en los niveles de desempeño alcanzado, y muy especialmente en las dimensiones léxica $(\mathrm{rho}=0.573 ; \mathrm{p}=0.032)$, semánti$\mathrm{ca}(\mathrm{rho}=0.680 ; \mathrm{p}=0.008)$ y pragmática $(\mathrm{rho}=0.553$; $\mathrm{p}=0.04$ ) (Rincón y Hederich, 2008: 57). Por otro lado, para el caso del grupo de método global, los estudiantes independientes de campo presentan unos desempeños levemente inferiores con relación a los demás. En este caso, sin embargo, las diferencias no alcanzan a ser estadísticamente significativas en las dimensiones de producción escrita formal (rho=0.205; $\mathrm{p}=0.399)$, semántica (rho=-0.212; $\mathrm{p}=0.383$ ), léxica $(\mathrm{rho}=-0.077 ; \mathrm{p}=0.755)$ y pragmática (rho=0.093; $\mathrm{p}=0.705$ ). En otras palabras, no sería posible afirmar que el método global privilegie a los sujetos sensibles al medio.

A fin de ofrecer una aproximación teórica acerca de la relación que guardan los métodos de enseñanza y los estilos cognitivos para explicar el logro de aprendizaje, se retoman los aportes de Bialystok (1992), quien explica que para la realización de toda tarea de orden lingüístico, se requiere de dos componentes esenciales de la habilidad metalingüística, en los que se hallarían procesos ejecutados por el niño, que estarían relacionados con las tareas requeridas para medir la DIC. El primer componente se denomina “análisis del conocimiento lingüístico", referido al proceso por el cual las representaciones mentales implícitas son reorganizadas de modo que alcancen el nivel de estructuras de representación explícita. Este mismo proceso ha sido descrito por Karmiloff Smith (1992) como redescripción representacional, en la que "los niños pequeños superan su maestría conductual, es decir, su fluidez verbal y éxito en la tarea comunicativa, para explorar el conocimiento lingüístico que tiene almacenado". En el proceso de aprendizaje del código escrito, este componente podría evidenciarse en el momento en el que los niños son capaces de autocorregirse, reconociendo, por ejemplo, la división correcta de las palabras, porque ya son conscientes que estas se separan por su significado, su función sintáctica y semántica dentro de las oraciones. En otras palabras, desarrollan conciencia metalingüística.

El segundo componente se denomina "el control del proceso lingüístico” que vincula la atención selectiva como aspecto fundamental, ya que a través de esta es posible atender las representaciones necesarias para la resolución de problemas lingüísticos. Un mayor desarrollo de control sobre la atención permite al niño funcionar bajo condiciones distractoras o ambiguas y distinguir entre información relevante y no relevante." (Rincón, 2008). Es este último componente, el que guarda una relación con las tareas de medición de la DIC, como el CEFT (Children Embedded Figures Test). A este respecto, Bialystok plantea que tanto los procesos metalingüísticos como los procesos implicados en la DIC requieren que la atención sea enfocada en un aspecto de la información de entrada, normalmente con la presencia de información sobresaliente desde un aspecto irrelevante. En este caso, el mecanismo encargado de la solución de problemas metalingüísticos es el control del proceso de atención, que podría ser entendido como un mecanismo de dominio general, que de igual forma estaría implicado en la resolución de problemas que no corresponden al campo lingüístico; en este caso, se refiere a las tareas de identificación de DIC.

A partir de este enfoque planteado por Bialystok, el estudio de Rincón y Hederich (2008) presenta un 
análisis de las posibles relaciones que cada uno de los métodos para la enseñanza de la escritura establece con los componentes de la habilidad metalingüística, para explicar por qué los métodos podrían estar favoreciendo a ciertos individuos sobre otros, en su logro de aprendizaje, a partir de sus características individuales.

Así, se dice que el método global guarda una relación directa con procesos metalingüísticos asociados al análisis del conocimiento lingüístico, en el que se le asigna al sujeto un papel central en el aprendizaje, ya que este implica un recorrido a través de diferentes habilidades lingüísticas que van desde lo conversacional, pasando por un conocimiento del código, para finalmente llegar a un grado de desarrollo que lo conduzcan a reorganizar y redescribir las representaciones de dicho conocimiento, lo que implica hacer los saberes de dominio del código más explícitos e integrarlos con otros.

Por esta razón, el método global respeta el proceso propio de la "evolución de la alfabetización" a partir de las experiencias de los niños, lo que les permite, al tiempo que representan, construyen y comprueban sus hipótesis lingüísticas, establecer ciertas trayectorias que dan forma al desarrollo de las competencias lingüísticas, lo que a su vez implica una prefiguración de la función social del lenguaje. Asimismo, aunque el control del proceso, si bien no es lo más importante en este método, sí se evidencia en el desarrollo, en el momento en que los sujetos logran establecer el valor de correspondencia entre fonema-grafía, que con el tiempo lo conducirá a un nivel de "conciencia fonológica".

Ahora bien, tomando la propuesta de Bialystok desde la perspectiva del método silábico, el componente de control de proceso toma relevancia, en tanto demanda de los sujetos un nivel de discriminación entre los elementos relevantes y aquellos que no lo son, es decir, se parte de la capacidad del sujeto para apropiar reglas de construcción de un código escrito que se valida dentro de un contexto de comunicación específico, lo cual se aplica a través de la "enseñanza sistemática de las reglas de conversión grafema-fonema [...] lo que significa que el niño tiene que ser capaz de segmentar las palabras en sus letras componentes y de asignar a cada letra el sonido que le corresponde" (Cuetos, 1996, 99). En esta dirección, son los sujetos independientes de campo quienes se encuentran en ventaja, dado que tienen un mayor desarrollo del control de la atención, lo que les hace más eficaces en la resolución de tareas metalingüísticas, automatizando en poco tiempo los procesos de conciencia fonológica para avanzar a niveles más complejos de producción escrita.

En términos generales, el estudio señala cómo los estudiantes en cuyo desarrollo perceptual hay un mayor predominio hacia la independencia de campo, tienen una facilidad para acceder a la comprensión del código lingüístico desde sus diferentes planos, estando en capacidad de aislarlo de su contexto de uso, privilegiando un mayor control de los procesos de correspondencia grafía-fonema. Tal proceso implica a su vez mayores niveles de reestructuración del sistema de la lengua en tanto demanda análisis y comprensión de todas y cada una de las unidades del código escrito, así como las relaciones que se establecen entre ellas, lo que favorece el uso de un método silábico entre este grupo de sujetos.

En el caso de los estudiantes que aprendían bajo las estrategias del método global, se evidenció una leve ventaja, no significativa, por parte de aquellos con una tendencia a la sensibilidad al medio. Sin embargo, se hace necesario aclarar que los grupos no eran totalmente comparables, en la medida que parte de la muestra se encontraba en preescolar $(58 \%)$ y la restante (42\%) en primero, lo que plantea una diferencia en términos de la maduración cognitiva y posiblemente también en relación con exposiciones al uso y manejo formal de elementos de la lengua escrita, y aun en estas condiciones es posible avizorar que es el método global el que ofrece unos desempeños similares para ambos grupos.

En conclusión, aunque los resultados de la investigación no pudieron determinar de forma concluyente que un método sea definitivamente mejor que otro, es posible decir, en primer lugar, que el método global tiende a un equilibrio en función del desarrollo de los procesos de aprendizaje del código escrito para sujetos con diferencias individuales, y en segundo lugar, que ante determinadas orientaciones 
metodológicas, los sujetos independientes de campo desarrollarían estrategias de adaptación frente a un contexto de aprendizaje, permitiéndoles tener éxito en el logro.

Es importante señalar que el estudio de Rincón y Hederich (2008) sugería para futuras investigaciones establecer análisis entre grupos que pudiesen ser comparables, dado que la muestra de la población con la que se trabajó era de estudiantes de dos niveles de educación básica distintos (preescolar y primero), con edades distintas, lo que impedía establecer una comparación entre estos. En esta medida, el presente estudio proporciona más información ya que trabaja con una muestra de estudiantes del mismo nivel de escolaridad, con niños de edades semejantes, aunque de instituciones diferentes.

\section{Metodología}

\section{Muestra}

Se seleccionó una muestra constituida por 31 niños (15 varones y 16 mujeres), de edades comprendidas entre los 5 y los 7 años $(M=6,35$; $D T=0,55)$ que se encontraban cursando primer grado de primaria en dos instituciones distintas con métodos diferentes: 16 sujetos bajo el método silábico y 15 sujetos bajo el método global. Cada una de las instituciones siguió un método diferente, por lo que no es posible separar las variables institución y método en este diseño. Al inicio del experimento los niños participantes ya han cursado el grado de transición en el que han tenido una experiencia previa de acercamiento al código escrito, de modo que la aplicación del pretest se da un año después de que los niños han accedido a la educación formal y han recibido formación con respecto a la escritura y la lectura.

\section{Diseño}

El estudio se inscribe dentro de un modelo analítico- explicativo, en tanto busca hallar una relación entre una variable independiente (método para la enseñanza de la lengua escrita), y una variable dependiente (logro en el aprendizaje de la lengua escrita), considerando como variable asociada el estilo cognitivo en la dimensión DIC.
En términos formales, la investigación sigue un diseño descriptivo comparativo que da cuenta del proceso de aprendizaje de la escritura en dos circunstancias diferentes, caracterizadas por dos métodos de enseñanza (silábico y global). Además, se explora el efecto de la variable de estilo cognitivo, en la dimensión dependencia - independencia de campo, sobre el logro de aprendizaje en las dos circunstancias anotadas. Se aplicó el instrumento para la determinación del nivel de logro en escritura en dos momentos: uno, al inicio de la observación de la metodología de enseñanza, y otro a 6 meses de iniciada la observación.

\section{Variables}

La variable independiente corresponde al método de enseñanza de escritura (silábico y global). Se definió como la variable dependiente el nivel de logro del aprendizaje en la lengua escrita, medida según el desarrollo de ocho indicadores de producción escrita: Uso de grafías convencionales, uso de reglas de combinación, uso de letra claramente distinguible, secuencia temática, coherencia textual, uso de no palabras, uso de elementos de conjunción y expresión narrativa. Finalmente, la variable asociada es el estilo cognitivo, en la dimensión de dependencia - independencia de campo (DIC).

\section{Instrumentos}

La prueba CEFT (Children embedded figures test) para identificar estilo cognitivo. Esta es una versión del Test de Figuras enmascaradas (EFT) diseñada para niños. Esta prueba mide la capacidad de reestructuración perceptual en niños menores de 9 años. La primera parte de la prueba consiste en hallar una figura simple en 25 tarjetas donde hay imágenes complejas. Así, el máximo puntaje que se puede obtener es 25 .

El pre - postest de escritura "Ejercicio de creación de texto": en esta prueba los niños tienen que producir un texto pequeño con ciertas características específicas. Para ello, se les plantea una situación particular enmarcada dentro de un contexto comunicativo con propósito; así, el niño redacta un escrito donde dé cuenta de su nivel de producción escrita. En este 
caso, la docente cuenta una historia (puede ser de literatura infantil), pero sin dar a conocer el final o desenlace, pues este es inventado por cada uno de los niños, según su creatividad. La medición de este test se da a través de una rejilla que propone ocho indicadores de producción escrita, diseñados a partir de cuatro dimensiones asociadas a estos:

\begin{tabular}{|c|c|}
\hline Indicadores & Dimensión \\
\hline $\begin{array}{l}\text { Uso de grafías convencionales/ perte- } \\
\text { necientes al alfabeto español }\end{array}$ & \multirow[t]{3}{*}{ Dimensión formal } \\
\hline $\begin{array}{l}\text { Sigue las reglas de posibilidad de } \\
\text { combinación }\end{array}$ & \\
\hline Cada letra es claramente distinguible & \\
\hline Sigue una secuencia temática & \multirow[t]{2}{*}{ Dimensión semántica } \\
\hline Coherencia textual & \\
\hline Uso de no palabras & \multirow{2}{*}{$\begin{array}{l}\text { Dimensión léxico - } \\
\text { sintáctica }\end{array}$} \\
\hline $\begin{array}{l}\text { Emplea elementos de conjunción, artí- } \\
\text { culos, preposiciones, etc. }\end{array}$ & \\
\hline $\begin{array}{l}\text { Responde a los requerimientos prag- } \\
\text { máticos de la situación, es decir, re- } \\
\text { fleja los rasgos de un texto narrativo }\end{array}$ & Dimensión pragmática \\
\hline
\end{tabular}

El examen de los niveles de consistencia interna del instrumento de escritura muestra altos niveles de confiabilidad, superiores a los corrientemente aceptados, indicados por un valor del alfa de Cronbach de 0.907, para 8 ítems.

\section{Procedimiento}

Los datos se obtuvieron de la aplicación de instrumentos en dos cursos de primer grado de dos instituciones educativas en Bogotá. En cada uno de los cursos, las docentes titulares desarrollaron una propuesta de formación en español orientada a partir de un método particular. Para el caso de una institución, el método seleccionado fue el silábico y para la otra, el método global. Para el inicio de recolección de datos, se aplicaron dos pruebas en cada grupo: la prueba CEFT para medir el estilo cognitivo y la prueba de escritura. Luego, se hizo un seguimiento a través de la observación directa de las clases, durante un periodo de cuatro meses, a fin de documentar, de forma más precisa, las características del método de enseñanza. Finalizado este tiempo de observación, se aplicó de nuevo la prueba de escritura.
Los puntajes de CEFT permitieron establecer los tres grupos de estilo cognitivo (sensibles, intermedios e independientes) por su ubicación en el primero, segundo o tercer tercil del puntaje CEFT. Sujetos cuya puntuación en el CEFT estuvo por debajo de 8 puntos, se agruparon en la categoría de "sensibles", sujetos con una puntuación entre 9 y 14 , se agruparon en la categoría de "intermedios" y sujetos con una puntuación superior a 15 , se agruparon en la categoría de "independientes".

La medición del pre y postest se hizo por parte de tres evaluadores, a partir de los indicadores de producción escrita establecidos. Cada indicador fue valorado a través de una escala de gradación en la que 1 correspondía a bajo, 2 a medio y 3 a alto. Con el propósito de unir los puntajes de los tres evaluadores se examinaron las correlaciones no parámetricas de Spearman entre las evaluaciones hechas para cada indicador de producción escrita. Las correlaciones presentadas entre los evaluadores oscilan entre 0.49 en el más bajo y 0.97 en el más alto, mostrando altos niveles de significación en todos los casos. Dadas estas altas correlaciones, se calculó el promedio de los puntajes de los evaluadores y se asumió tal promedio como indicador de desempeño en cada uno de los indicadores de la prueba.

Para revisar los efectos de la variable independiente, la asociada y su interacción, sobre la variable dependiente, se aplicó un análisis univariado de covarianza. El análisis permitió evaluar de manera independiente el efecto del método en cada uno de los indicadores de medición del postest (uso de grafías convencionales, uso de reglas de combinación, uso de letra claramente distinguible, secuencia temática, coherencia textual, uso de no palabras, uso de elementos de conjunción y expresión narrativa), controlando los niveles previos.

\section{Resultados}

La gráfica muestra los promedios de los tres evaluadores frente a los ocho indicadores de producción escrita, medidos en la primera prueba de escritura, diferenciados por método o, lo que es lo mismo, por grupo escolar. 
Tal y como se observa, las diferencias entre los dos grupos son amplias. Solo en dos de los ocho indicadores considerados las diferencias en las condiciones iniciales de los grupos no son estadísticamente significativas: el uso de grafías convencionales ( $t=1.41 ; \mathrm{p}=0.167)$, $\mathrm{y}$ en el uso de reglas de combinación ( $t=1.28 ; \mathrm{p}=0.212$ ). En todos los demás indicadores las diferencias en las condiciones iniciales son altamente significativas a favor del grupo que ha venido utilizando el método global, y muy particularmente en coherencia $(\mathrm{t}=9.74, \mathrm{p}<0.001)$, elementos de conjunción ( $\mathrm{t}=8.43, \mathrm{p}<0.001)$, narrativa $(\mathrm{t}=8.01, \mathrm{p}<0.001)$ y secuencia temática $(\mathrm{t}=5.09$, $\mathrm{p}<0.001)$. Con niveles de significación levemente menores se observan diferencias en uso de no palabras $(\mathrm{t}=3.55, \mathrm{p}=0.001)$ y uso de caligrafía clara $(\mathrm{t}=3.38, \mathrm{p}=0.002)$. Ver gráfica 1 .

Gráfico 1: Frecuencia de sugerencia de formato de evaluación docente

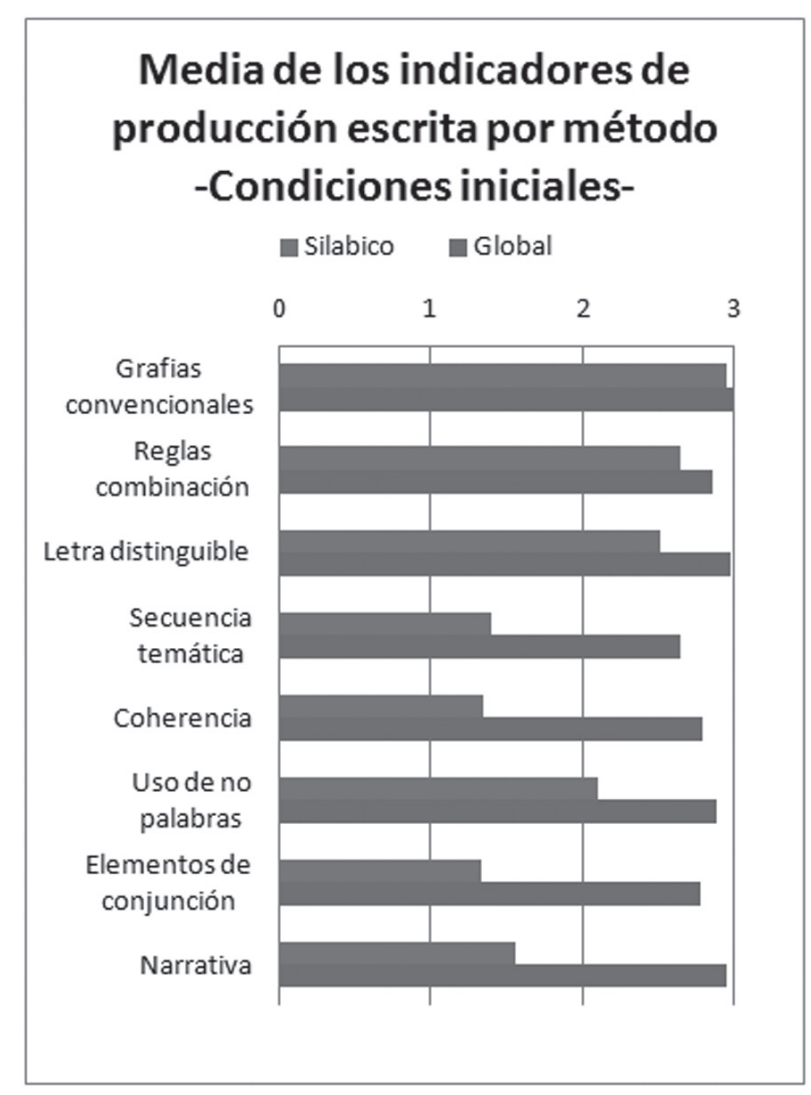

Los bajos niveles de significación demostrados en la diferencia de los estados iniciales de los grupos en los indicadores del uso de grafías convencionales y en el uso de reglas de combinación están asociados al hecho de que ambos, como descriptores de la dimensión formal del desarrollo de producción escrita, son elementos esenciales en los dos métodos. Dicho de otro modo, tanto el método silábico como el global, se plantean como parte de sus principales objetivos lograr que los niños reconozcan y empleen correctamente las grafías que representan cada letra del alfabeto y que hagan combinaciones entre estas de acuerdo con las reglas de la lengua objeto de aprendizaje. De este modo, el método silábico dedica un amplio porcentaje del desarrollo del curso al aprendizaje de la correspondencia entre el fonema y la grafía y la existencia de un número limitado de combinaciones, a través del reconocimiento de las sílabas. No obstante, el método global supera al silábico en el desarrollo asociado a otras dimensiones de nivel superior, desde la perspectiva psicolingüística, como el conocimiento semántico, sintáctico, lexical y pragmático. Esto se refleja en los niveles de significación encontrados al examinar las diferencias en los otros seis indicadores, en donde hay una clara ventaja por parte de los sujetos que hacen parte del grupo donde se enseña el método global.

La tabla 1 presenta los resultados sintéticos de los ocho análisis de covarianza, examinados para la predicción de cada uno de los indicadores de producción escrita a partir del grupo (variable en la que se juntan el método y la institución), el estilo cognitivo y la interacción entre grupo y estilo. Estos niveles de logro se controlan por covariables definidas como los estados iniciales de cada uno de los indicadores.

Tal y como se observa, todos los modelos obtenidos muestran niveles de predicción aceptables, algunos muy notables, que se diferencian de forma significativa en su capacidad predictiva del azar. Se destacan el modelo para la predicción del indicador de uso de letra distinguible $(\mathrm{F}=11.75, \mathrm{p}<0.001)$ que alcanzan a predecir el $68.2 \%$ de la varianza, y en donde todos los factores y sus interacciones muestran asociaciones significativas, y el uso de no palabras $(\mathrm{F}=8.28, \mathrm{p}<0.001)$, en donde se explica el $59 \%$ de la varianza a partir de la interacción entre grupo y estilo, el estilo como efecto principal, y, en menor medida, el grupo como efecto principal. 
Tabla 1. Análisis de covarianza de los ocho indicadores de producción escrita

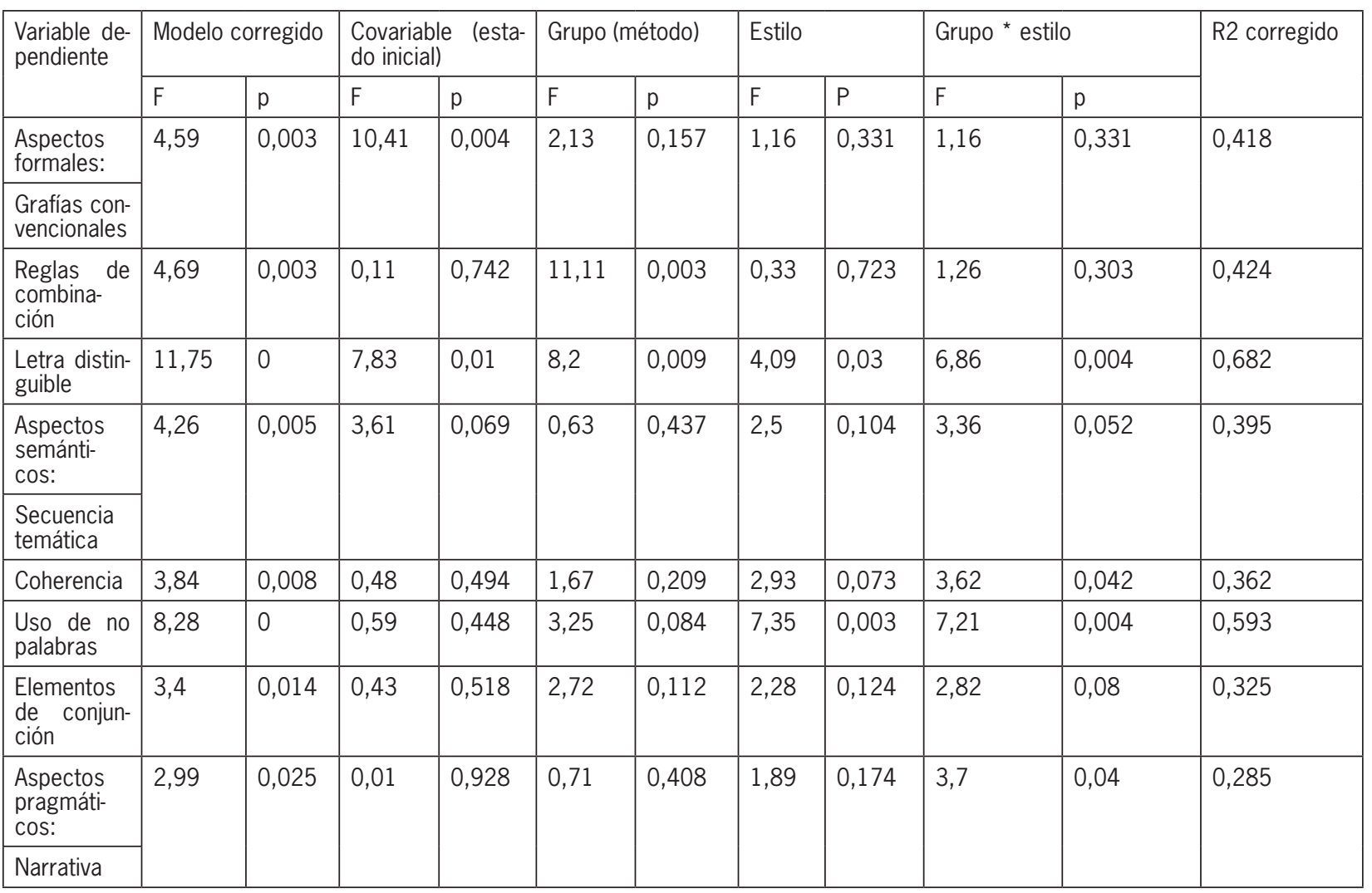

Ya en particular, las variables que mejor explican la varianza de los estados finales difieren de forma importante entre los modelos.

En términos generales, la variable que logra mayores niveles de asociación con los diferentes indicadores es la interacción entre grupo y estilo, que resulta con asociaciones apreciables en seis de los ocho modelos, y siempre en el mismo sentido: los sujetos más independientes se ven favorecidos por el uso del método silábico y los más sensibles se ven desfavorecidos por el mismo. Estas asociaciones resultan ser muy significativas $(\mathrm{p}<0.01)$ en dos de los modelos: uso de no palabras y letra distinguible, levemente significativas $(0.01<\mathrm{p}<0.05)$ en otros dos: coherencia y narrativa; $y$ con niveles muy cercanos a la significancia convencional $(0.05<\mathrm{p}<0.10)$ aparecen otros dos indicadores: secuencia temática y elementos de conjunción.

El grupo, en tanto efecto principal, variable en la que coinciden método (global/silábico) e institución, muestra asociaciones apreciables, en tres de los ocho modelos: el uso de reglas de combinación $(\mathrm{p}=0.003)$, la letra distinguible $(\mathrm{p}=0.009)$ y el uso de no palabras $(\mathrm{p}=0.084)$. La influencia del grupo aparece siempre en el mismo sentido: aquellos sujetos que trabajaron con el método global muestran mejores logros que los que trabajaron con el método silábico. Esta influencia se ve claramente en todos los modelos examinados.

En situación similar aparece la influencia del estilo, en tanto efecto principal, que también muestra asociaciones significativas en tres de los ocho modelos, siempre en el mismo sentido: los sujetos independientes muestran mejores logros, siendo seguidos por los sujetos intermedios y estos últimos por los sensibles. El uso de no palabras muestra asociaciones muy significativas ( $\mathrm{p}=0.003$ ) y la letra distinguible muestra asociaciones significativas $(\mathrm{p}=0.030)$; la coherencia, finalmente, muestra niveles cercanos a la significación convencionalmente aceptada ( $\mathrm{p}=0.073$ ). Incluso en los modelos en los que la asociación no resulta significativa, se observa la misma dirección de la influencia: los independientes obtienen mejores logros que los intermedios, 
y estos a su vez, obtienen mejores logros que los sensibles.

Las covariables muestran, en general, escasos niveles de explicación, salvo los casos de grafías convencionales $(\mathrm{F}=10.41, \mathrm{p}=0.004)$ y letra distinguible $(\mathrm{F}=7,83, \mathrm{p}=0.010)$. En todos los demás casos los estados iniciales de los indicadores no explican de forma significativa los estados finales en esos mismos indicadores.

La gráfica 2 presenta las medias marginales estimadas de cada uno de los indicadores de producción escrita en el postest, comparando los dos grupos. A través de la diferencia de medias se reitera lo que mostró el análisis de covarianza con relación a la variable de interacción entre grupo y estilo, en la que se haya que los sujetos más independientes de campo se ven favorecidos por el uso del método silábico, mientras que los más sensibles presentan una notoria desventaja en el mismo. Ver gráficas 2(a) a 2 (h).

En el caso del método silábico, los resultados obtenidos por los sujetos sensibles demuestran

Gráfica 2a: Medias marginales estimadas para grafías convencionales

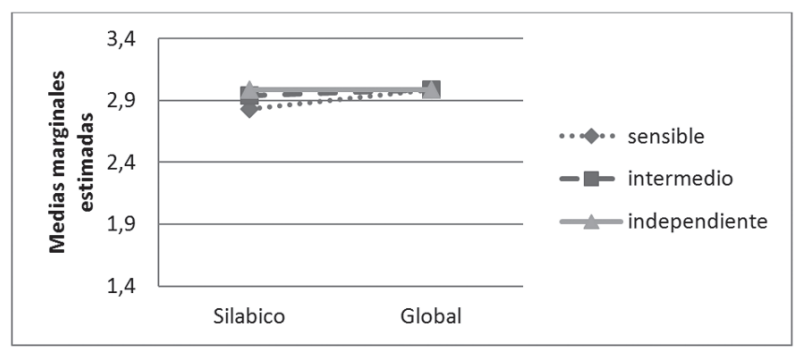

Gráfica 2c: Medias marginales estimadas para uso de letra claramente distinguible

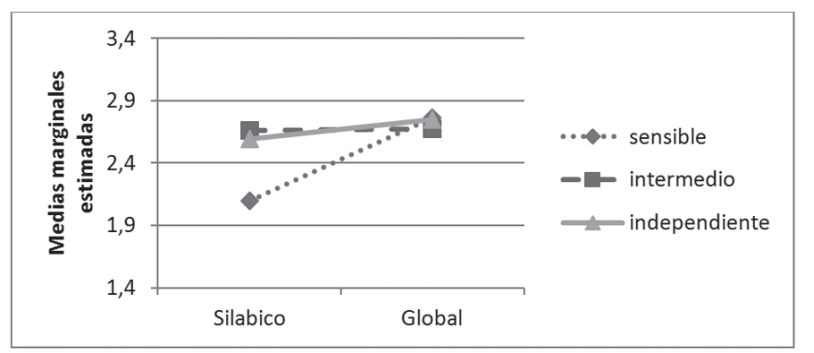

que estos siempre están por debajo de los resultados obtenidos por los intermedios y los independientes, poniéndolos en una notoria desventaja. Entre los intermedios e independientes no hay una diferencia muy amplia; sin embargo, se observa que en siete de los ocho indicadores, los independientes obtienen resultados superiores a los intermedios, la excepción se presenta en el indicador de "uso de secuencia temática".

En el caso del método global, los resultados de la interacción entre grupo y estilo señalan un comportamiento muy diferente. Para este grupo, no parece haber diferencias significativas entre los resultados de los sujetos independientes, intermedios y sensibles. Incluso, en tres de los ocho indicadores (uso de grafías convencionales, uso de secuencia temática y uso de no palabras) se muestra que los sujetos con estilos cognitivos distintos alcanzan el mismo nivel de logro (Gráfica 2.a, 2.d, 2.f). En los otros indicadores se observan ligeras diferencias, pero nada significativo como para afirmar que el método global ponga a cierto grupo de sujetos en ventaja o desventaja, de acuerdo a su estilo cognitivo.

Gráfica 2b: Medias marginales estimadas para uso de regla de combinación

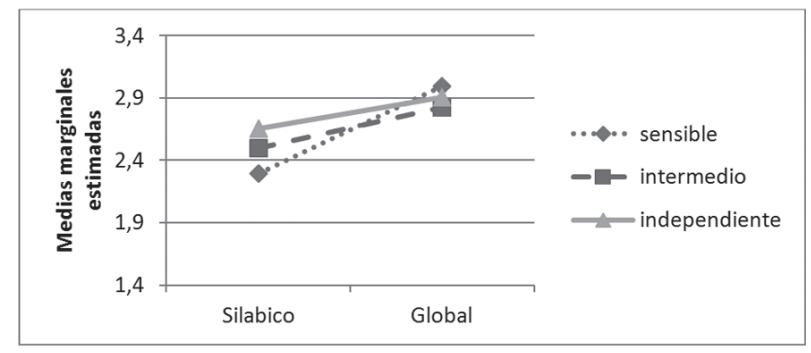

Gráfica 2d: Medias marginales estimadas para uso de secuencia temática

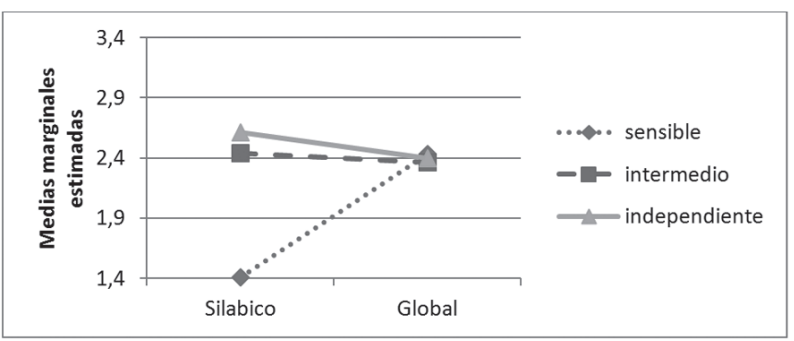


Gráfica 2e: Medias marginales estimadas para coherencia textual

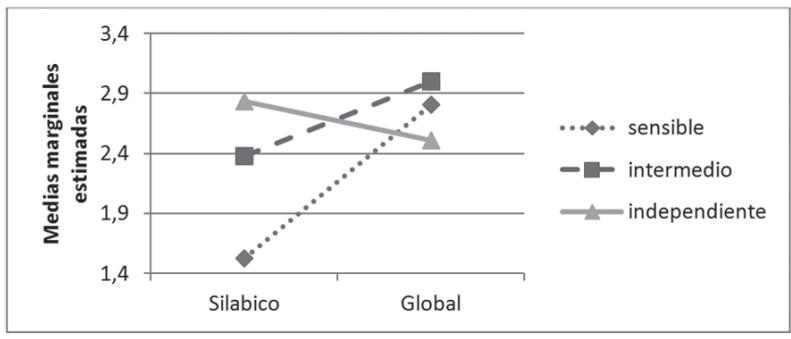

Gráfica 2g: Medias marginales estimadas para uso de elementos de conjunción

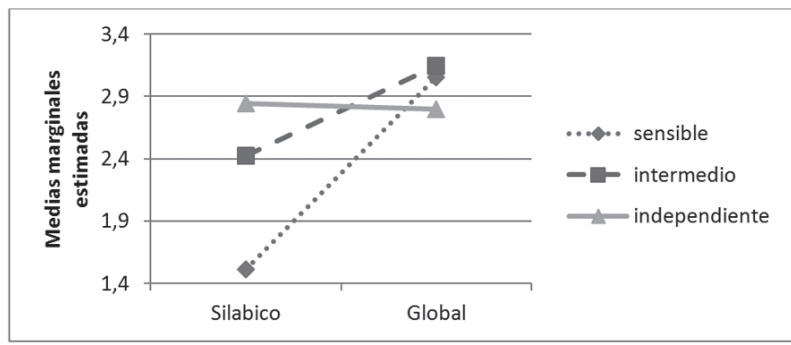

El estudio demuestra que, comparando ambos métodos, el global parece respetar las diferencias individuales y promover los logros de aprendizaje de forma equilibrada entre los sujetos con estilos cognitivos distintos. Además, al revisar los niveles de logro alcanzados por los niños, esto parece ser un factor determinante, pues para el caso del grupo que aprendió con el método global se demuestra que alcanzó niveles superiores a los alcanzados por los participantes del método silábico.

\section{Discusión y conclusiones}

Este estudio se diseñó con el fin de explorar el efecto diferencial de la implementación de los métodos silábico y global para la enseñanza de la escritura, sobre el logro de aprendizaje en niños con estilos cognitivos diferentes en la dimensión de dependencia-independencia de campo. Al respecto, los antecedentes presentados por Rincón y Hederich (2008) señalan que el método silábico, por su carácter sintético, es decir, que inicia el aprendizaje a partir del estudio de signos aislados de la lengua (grafías) y su correspondencia con los fonemas, favorece más a los sujetos independientes de campo, dada su facilidad para reestructurar información y
Gráfica 2f: Medias marginales estimadas para uso de no palabras

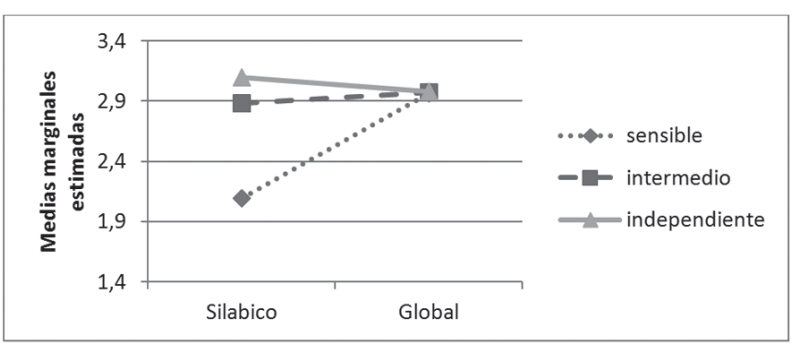

Gráfica 2h: Medias marginales estimadas para narrativa

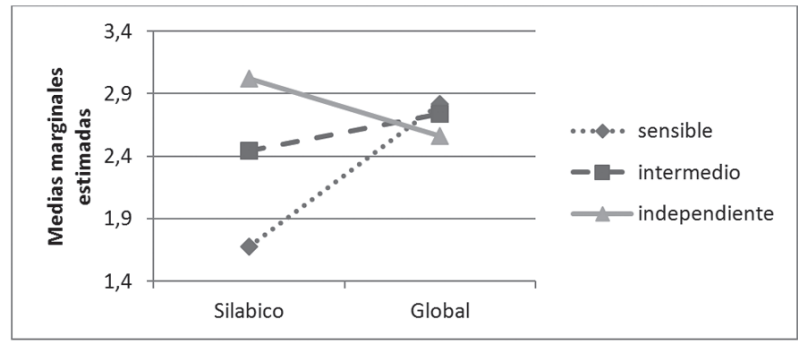

para resolver problemas que requieren sacar los elementos de su contexto general para ser procesados. Además, se señala la relativa ecuanimidad del método global al no demostrar diferencias significativas en el logro de aprendizaje entre los individuos de estilos cognitivos diferentes. Sin embargo, tal estudio había mencionado la necesidad de comparar grupos que pertenecieran al mismo nivel de escolaridad, con niños cuyas edades fueran semejantes.

El presente estudio, realizado con poblaciones en el mismo grado de escolaridad, demostró que el método silábico beneficia a los sujetos independientes, y en cierta medida a los intermedios; a pesar de esto, a los sensibles los deja en una notoria desventaja. El nivel de logro que pudiese alcanzar el sujeto sensible es significativamente bajo en comparación con independientes e intermedios. Frente a esta situación, Rincón y Hederich (2008) explicaron que el alto desempeño de los independientes en las tareas demandadas por el método silábico podría estar asociado al concepto de "control de atención" que ya había sido explorado por Bialystok (1992). En ese sentido, los sujetos que demuestran tener un alto control de atención, resultan ser muy hábiles en las tareas que involucran la eliminación de aspectos 
irrelevantes y distractores durante el procesamiento de la información. Así, al resolver la prueba del CEFT, estos sujetos tendrían altos puntajes, lo que demostraría que tienen una alta capacidad de reestructuración perceptual. Lo mismo ocurriría en las tareas de escritura propuestas por el método silábico, en las que tendrían un alto desempeño en todo lo que requiera sacar los elementos de su contexto completo, dicho de otro modo, sacar las grafías del contexto de las palabras y procesarlas como elementos aislados que se relacionan con otros elementos aislados llamados fonemas.

Guisande, Páramo, Tinajero y Almeida (2007) explican que los procesos de atención son de gran influencia en niños con edades tempranas y es precisamente el estilo cognitivo el que se convierte en el factor determinante. Según sus estudios, se encontró que los niños independientes parecen ser quienes aprovechan mejor su capacidad atencional. Mientras que los niños sensibles en el momento de resolver la tarea se encuentran saturados con la información presentada en el campo perceptual, lo que impediría el uso efectivo de su capacidad operativa. De este modo, su desempeño se vería muy por debajo de su verdadero potencial.

Esta explicación se sustenta en el modelo de dialéctico de Pascual-Leone (1987) y su teoría de los operadores constructivos, en la que se define la capacidad de atención mental como "el recurso endógeno de atención que permite a los sujetos tener en cuenta, a través de la activación endógena de regímenes correspondientes, un determinado número de características de la tarea o las restricciones que no están directamente activadas por la situación perceptiva" (Pascual-Leone, Baillargeo y Roncadin, 1998: 145). En este sentido, tanto niños sensibles como independientes, de edades semejantes, tendrían la misma capacidad de atención mental; no obstante, los sujetos sensibles carecerían de sistemas sofisticados de ejecución para focalizar la capacidad de atención de forma adecuada, especialmente, frente a tareas donde hay información distractora o engañosa.

Frente al caso del aprendizaje de la escritura, habría que mencionar que desde el método silábico se le exige al niño seleccionar información relevante dentro de mucha información que opera en ciertos momentos como distractora. Esto se da cuando el niño debe sacar la unidad mínima que se aprenderá (la grafía) de un contexto más complejo (la palabra) para ser relacionada con la información fonético fonológica que ya conoce. Más adelante se le pedirá que asocie al menos dos de esas unidades mínimas aprendidas y sus sonidos correspondientes para formar sílabas. En principio, las tareas acá definidas estarían demandando de una capacidad de atención mental alta; en otras palabras, los niños tendrían toda su capacidad atencional saturada en estas demandas. Sin embargo, en el momento en que se logra automatizar la tarea de decodificación de grafías esta saturación disminuye, lo que implica que se libere capacidad atencional, así, quienes logren este nivel de automatización, estarán listos para avanzar en el proceso hacia la recuperación de significados, ya no procesando la información por separado, sino accediendo a la palabra como unidad.

Visto desde esta perspectiva, es claro que los sujetos independientes son más hábiles a la hora de resolver tareas con presencia de información distractora o engañosa, debido al uso de esquemas ejecutivos sofisticados, responsables de mover y asignar los recursos de atención mental al niño (Pascual-Leone, Baillargeo y Roncadin, 1998). Así, los niños independientes de campo tardan menos tiempo en automatizar las tareas de decodificación y correspondencia entre grafía - fonema, lo que les permite avanzar más rápidamente en el aprendizaje de aspectos de orden lexical, sintáctico, semántico y pragmático en la escritura y tomar una ventaja notoria sobre los niños con tendencia a la sensibilidad.

Por su parte, los niños sensibles carecen de los esquemas sofisticados de ejecución, por lo que se ven en la necesidad de reducir su posibilidad de obtener éxito en estas tareas de focalización de atención y de selección de información relevante sobre la irrelevante en situaciones confusas. Por esta razón, los niños sensibles que aprenden con el método silábico demoran más tiempo en automatizar los procesos de decodificación. Entonces, buena parte del curso lo invierten en mecanizar cada grafía, asociarla con su 
correspondiente sonido y comprender las relaciones posibles entre estas para formar sílabas; pero aún tardan mucho más en llegar a los niveles de producción desde lo sintáctico, semántico y pragmático.

En cuanto a los sujetos intermedios, cabe señalar que su relativa movilidad les permite acercarse a los independientes a la hora de seleccionar esquemas ejecutivos de capacidad de atención, lo que los ubica en un nivel de logro superior a los sensibles; no obstante, de acuerdo con los indicadores de producción escrita examinados, no podría afirmarse que están en las mismas condiciones de los independientes, pero si más próximos al nivel alcanzado por estos.

Tsakanikos (2006) señala otro factor que resulta relevante para comprender los resultados en el desempeño de los sujetos sensibles y los independientes en el aprendizaje a través del método silábico, dado por la tendencia al procesamiento analítico en el independiente. En efecto, durante el aprendizaje asociativo, si se presentan dos o más elementos de forma simultánea, el logro de aprendizaje depende de la tendencia a percibir cada elemento por separado, como una unidad, es decir, analíticamente. Desde este enfoque, también los sujetos independientes obtendrían mejores resultados que los sensibles, dado que a los independientes se les caracteriza como sujetos que experimentan su entorno analíticamente, separando los elementos de su contexto. Este tipo de procesos se requiere justamente en el método silábico.

Con respecto a los resultados encontrados en el grupo correspondiente al método global, es importante anotar que no se presentaron diferencias significativas entre el nivel de logro alcanzado por sujetos con estilo cognitivo diferente. Esto significa, a diferencia de lo hallado en el método silábico, que los independientes no estuvieron por encima de los sensibles, ni viceversa. Hay varios elementos que permitirían explicar la relativa ecuanimidad de este método y la posibilidad que le ofrece al sensible de obtener resultados tan buenos como los de los independientes.

En primer lugar, hay que mencionar que el método global se encuentra apoyado en un enfoque cooperativo de aprendizaje, en el que los sujetos actúan como reguladores del aprendizaje los unos de los otros. Es decir que los procesos se dan en un contexto en el que los mismos compañeros cooperan en las tareas de producción y corrección de sus escritos. Esto evidentemente favorece el desempeño de los sujetos sensibles, en tanto se caracterizan por una mayor orientación social y una preferencia por el trabajo en grupo (Chen y Macredie, 2002 y Lee et al, 2004). Tal cualidad les estaría permitiendo obtener soporte del medio para desarrollar las tareas que se le demanden, como la de escritura colectiva.

Por otro lado, debe discutirse el concepto de la globalización mencionado en la introducción. A partir de este concepto, se sostiene que los niños se caracterizan por percibir las cosas en conjunto (Braslavsky, 1962); tal cualidad es inherente a su desarrollo cognitivo natural. De este modo, todo niño que inicia el proceso de aprendizaje del código escrito a través del método global, lo hace a partir del conocimiento de su lengua oral, por un lado, y por otro, de las estrategias que le han permitido adquirirlo. Así, el niño tratará de acercarse al código escrito a través de una percepción global, es decir, de la comprensión del todo y no de sus partes constitutivas, tal y como lo hace cuando aprende conceptos (Karmiloff-Smith 1994 y Markman, 1990).

Esto favorece notoriamente el proceso de los sujetos sensibles al medio, permitiendo que empleen sus estrategias de procesamiento cognitivo características: experimentar su entorno de una manera relativamente global, conformes pasivamente a la influencia del campo o contexto dominante (Chen y Macredie, 2002; Lee et al, 2004). Ese mismo concepto de globalidad es el que conduce a que los niños interactúen con la escritura a través de textos completos y con sentido, no a través de unidades aisladas como la grafía. En esta medida, al proporcionarle un contexto más significativo y menos abstracto al niño sensible, este encuentra mejores herramientas para comprender el código y avanzar en dimensiones de orden semántico y pragmático de la lengua, más que de orden formal.

Sin embargo, debe tenerse en cuenta que el método no favorece a los sensibles por encima de los 
independientes, sino que los ubica en un nivel de logro semejante. Esto significa que aunque el método global haga énfasis en el aprendizaje cooperativo, el trabajo grupal y la presentación de la información de manera global, no está provocando desventajas en el desempeño de los niños independientes. Por el contrario, podría decirse que el método también proporciona las herramientas necesarias para quelos niños con tendencia a la independencia alcancen sus metas. En este sentido, cabe anotar que los procesos desarrollados a través del método silábico también podrían verse involucrados en cierta medida en este método. Por ejemplo, el aprendizaje asociativo, que no se daría entre grafía y fonema, como en el caso del método silábico, sino entre palabras, imágenes y sentidos.

Para finalizar, debe mencionarse que los resultados expuestos en este estudio tienen claras implicaciones en la concepción de métodos y estrategias de enseñanza de la escritura en los años iniciales de escolaridad. Por largo tiempo se ha discutido la pertinencia de los métodos, pero pocas veces se

\section{Referencias}

Anula, A. (2002). El abecé de la psicolingüística. Madrid: Arco Libros.

Benveniste, C. (2002). La escritura, irreductible a un “código". En E. Ferreiro (Ed.), Relaciones de (in)dependencia entre oralidad y escritura. Madrid: Gedisa.

Belinchón, M., Igoa, J. y Riviere, A. (2000). Psicología del lenguaje. Investigación y teoría. Madrid: Editorial Trotta.

Best, J. (2000). Psicología Cognitiva. Bogotá: McGrawHill.

Bialystok, E. (1992). Attentional Control in Children's Metalinguistic Performance and measures of Field Independence. En Developmental Psychology, Vol. 2, (No. 4), 654-664.

Braslavsky, B. (1962). La querella de los métodos en la enseñanza de la lectura. Sus fundamentos psicológicos y la renovación actual. Buenos Aires: Kapelusz. han explorado variables como las estudiadas acá, que buscan relacionar las diferencias individuales de los sujetos con sus modos de aprender y de procesar la información. Es claro que, aunque el método silábico funcione y haya sido aplicado por años dentro de las escuelas, no está considerando las características individuales de los estudiantes, dejando así, un buen número de niños en desventaja y ampliando significativamente la brecha entre el nivel de logro alcanzado por unos y otros. Por su parte, el método global, también empleado por largos años, al parecer resulta mucho más equilibrado al revisar el logro alcanzado por niños con estilos cognitivos diferentes. En esa medida, este último resultaría ser una mejor opción para que sujetos con diferencias individuales aprendan y alcancen la meta en todas las dimensiones de producción escrita que todo individuo en este nivel escolar debiera lograr. No obstante, para estudios futuros se requiere tomar poblaciones en condiciones similares de acuerdo a su contexto social y a las condiciones de la institución en la que están estudiando.

Cuetos, F. (1996). La psicología de la lectura. Barcelona: Editorial Praxis.

Ferreiro, E. (1983). Psicogénesis de la escritura. En C. Coll (comp), Psicología Genética y aprendizajes escolares. Madrid: siglo XXI.

Giraldo, O., Pérez, C. y Rincón, L. (2011). Informe final de investigación: Relaciones entre el aprendizaje de la lengua escrita, métodos de enseñanza y estilos cognitivos. Bogotá: Universidad Pedagógica Nacional/ CIUP.

Guisande, M., Páramo, F., Tinajero, C. y Almeida, L. (2007). Field dependence independence (FDI) cognitive style: An analysis of attentional functioning. En Psicothema, Vol. 19 (No. 4), 572-577.

Hederich, C. (2007). Estilos cognitivos en la dimensión de independencia-dependencia de campo: influencias culturales e implicaciones para la educación. Tesis Doctoral de la Universidad Autónoma de Barcelona. 
Colección Tesis Doctorales, 4. Universidad Pedagógica Nacional.

Hederich, C. y Camargo, A. (2001). Estilos Cognitivos en el contexto escolar. Proyecto de Estilos Cognitivos y logro educativo en la ciudad de Bogotá. Bogotá: Universidad Pedagógica Nacional. Centro de Investigaciones -CIUP. Instituto para la Investigación Educativa y el Desarrollo Pedagógico -IDEP.

Jollibert, J. (1991). Formar niños productores de texto. Chile: Editorial Dolmen.

Karmiloff-Smith, A. (1994). Mas allá de la modularidad. Madrid: Alianza.

Lee, C., Sudweeks, F., Cheng, Y. y Tang, F. (2010). The role of unit evaluation, learning and culture dimensions related to students cognitive style in hypermedia learning. En Proceedings Cultural Attitudes Towards communication and Technology. Australia: Murdoch University.

López, J. y Álvarez, J. (1991). Enseñanza de la lectura y psicología: Análisis de los "métodos" sintéticos. En Psicothema, Vol. 3 (No. 1), 121-131.

Markman, E. (1990). Constraints children place on word meanings. En Cognitive Science (No. 14), 57-77.

Morton, J. (1979). Word Recognition. En J. Morton y J. C. Marshall (Eds.), Psycholinguistics Series 2: Structures and processes. Londres: Elek.

Pascual-Leone, J., Baillargeo, R. y Roncadin, C. (1998). Mental-Attentional Capacity: Does Cognitive Style Make a Difference? En Journal of Experimental Child Psychology, 70, 143-166.

Rincón, L. (2008). Relaciones entre aprendizaje de la lengua escrita, métodos de enseñanza y estilo cognitivo. Bogotá: Tesis para aplicar al grado de Magister en Educación en la Universidad Pedagógica Nacional. Documento de circulación restringida.

Rincón, L. y Hederich, C. (2008). Relaciones entre aprendizaje inicial de la lengua escrita, métodos de enseñanza y estilo cognitivo. En Folios (No. 28), Julio-Diciembre, 51-63.

Solsona, J., Navarro, J, y Aguilar, M. (2009). La atención mental en el aprendizaje de la lengua escrita. En International Journal of Psychology and Psychological Therapy, 9, 335-349.

Tsakanikos, E. (2006). Associative learning and perceptual style: Are associated events perceived analytically or as a whole? En Personality and Individual Differences, 40, 579-586. 